\title{
Marcumar- und Clopidogrel-Nachfolger versprechen großen Fortschritt
}

\author{
Dr. med. \\ Dirk Einecke \\ Chefredakteur
}

\section{SCHLAGANFALLPROPHYLAXE BEI VORHOFFLIMMERN}

\section{Neuer Thrombininhibitor ist Marcumar überlegen}

Patienten mit Vorhofflimmern und Risikofaktoren für einen Schlaganfall benötigen eine orale Antikoagulation mit Vitamin-K-Antagonisten wie Marcumar. Diese sind jedoch problematisch: Schmale therapeutische Breite, erhöhtes Blutungsrisiko sowie zahlreiche Interaktionen machen ständige Überwachung notwendig. Patienten verfehlen häufig den INRZielbereich, viele setzen die Behandlung wieder ab.

Nun endlich wird es endlich eine Alternative zu Marcumar geben: Dabigatran (Pradaxa ${ }^{\circledR}$ ), ein oral einzunehmender direkter Thrombininhibitor. Dieser erwies sich in der sog. RE-LY-Studie Marcumar als therapeutisch überlegen. RE-LY testete zwei Dosierungen von Dabigatran, das in Deutschland bereits in der Thromboseprophylaxe im Einsatz ist: Eine niedrigere
Dosierung von $2 \times 110 \mathrm{mg} / \mathrm{d}$ schützte in gleichem Maße vor Schlaganfällen und Embolien wie Marcumar (1,5\% vs. 1,7\% pro Jahr), ging aber mit signifikant weniger schweren Blutungen einher (2,7\%/Jahr vs. $3,4 \% / J a h r$, RR: $-20 \%, p=0,003)$. Die höhere Dosierung von 2 x $150 \mathrm{mg} / \mathrm{d}$ war signifikant wirksamer als Marcumar (Schlaganfall/Embolie 1,1\% vs. 1,7\% pro Jahr, RR: $-34 \%, p=0,001$ ) bei gleicher Rate schwerer Blutungen (3,1\% vs. 3,4\% pro Jahr, $p=0,31$ ). Beide Dabigatrandosierungen führten zu weniger intrakraniellen und lebensbedrohlichen Blutungen als Marcumar. RE-LY untersuchte $18113 \mathrm{~Pa}$ tienten, die in drei Gruppen im Schnitt zwei Jahre lang behandelt wurden.

Jahreskongress der European Society of Cardiology 2009, Barcelona; St. J. Connolly et al., N Engl J Med 2009;361, online 30.8.2009

\section{AKUTE KORONARSYNDROME}

\section{Neuer Thrombozytenhemmer lässt mehr Patienten überleben}

Patienten mit akuten Koronarsyndromen benötigen zwei Thrombozytenhemmer. Standard ist ASS plus Clopidogrel. Jetzt gibt es Alternativen zu Clopidogrel, das Nachteile aufweist, unter anderem einen langsamen Wirkbeginn und deutliche Schwankungen in der Wirksamkeit. Eine ist das kürzlich zugelassene Prasugrel (Efient $\left.{ }^{\circledR}\right)$, eine andere wird wohl Ticagrelor sein (vorgesehener Handelsname: Brilinta ${ }^{\circledR}$ ). Ticagrelor, oral einzunehmen, wirkt schneller und stärker als Clopidogrel und besitzt den Vorteil, dass es die ADP-Rezeptoren der Thrombozyten reversibel hemmt.

In der PLATO-Studie, vorgestellt beim europäischen Herzkongress, überraschte Ticagrelor mit positiven Ergebnissen: Im Vergleich zu Clopidogrel reduzierte es signifikant koronare Komplikationen (Koronartod, Infarkt, Schlaganfall, 9,8\%

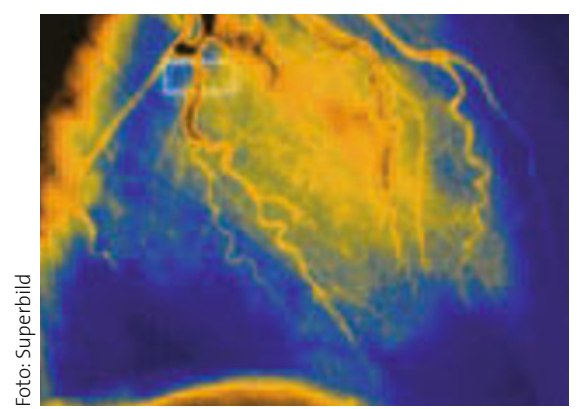

Bei akuten Koronarsyndromen ist Ticagrelor wirksamer als Clopidogrel.

vs. $11,7 \%, p<0,001)$, ohne die Blutungsrate zu erhöhen. In der Ticagrelorgruppe überlebten signifikant mehr Patienten (95,5\% vs. $94,1 \%, p<0,001)$. PLATO untersuchte 18624 Patienten.

Jahreskongress der European Society of Cardiology 2009, Barcelona; L. Wallentin et al., N Engl J Med 2009;361, online 30.8.2009

\section{NEUER REKORD}

\section{Patienten immer früher aus dem Krankenhaus entlassen}

Die durchschnittliche Dauer eines Krankenhausaufenthaltes ist in den letzten Jahren stark zurückgegangen. Während es im Jahr 1991 noch ganze 14 Tage waren, wurden die Patienten im vergangenen Jahr schon nach 8,1 Tagen wieder nach Hause geschickt - ein neuer Tiefstand. Insgesamt wurden 17,4 Millionen Menschen vollstationär behandelt, 200000 mehr als im Vorjahr. Für sie standen 2067 Krankenhäuser mit 500 ooo Betten zur Verfügung, 20 Krankenhäuser und 7500 Betten weniger als 2007. Die kurzen Verweildauern sind sicher auch eine ökonomisch motivierte Reaktion auf die Fallpauschalen. Den Preis dafür zahlen die niedergelassenen Ärzte, denen die Versorgung der frühzeitig entlassenen Patienten übertragen wird.

Pressemitteilung Statistisches Bundesamt, 2.9.2009

\section{BEHANDLUNGSFEHLER}

\section{Wem Patienten am ehesten verzeihen}

Gesteht ein Arzt einen Behandlungsfehler offen und ungeschminkt ein und entschuldigt sich dafür, wird ihm sein Patient eher weiter vertrauen, als wenn er die Wahrheit verschleiert und auf eine Entschuldigung verzichtet. Das ergab eine US-Studie, in der 200 Probanden Videos sahen, die den Umgang von Ärzten mit gravierenden Kunstfehlern zeigten. Die Filmärzte entschuldigten sich entweder gar nicht, unspezifisch oder ausführlich. Einige übernahmen die volle Verantwortung für den Fehler, andere nicht. Ehrlichkeit und Offenheit kommt gut an, so das Fazit der Studie. Allerdings würden die meisten Befragten auch die offenen, ehrlichen Ärzte auf Schmerzensgeld verklagen.

J General Int Med 2009;24(9):1012 